\title{
Mayer, Marius (2013): Kosten und Nutzen des Nationalparks Bayerischer Wald. Eine ökonomische Bewertung unter Berücksichtigung von Tourismus und Forstwirtschaft
}

\author{
München - oekom verlag. 107 Tab., 55 Abb., 575 S.
}

\section{Ulrich Hampicke}

(C) Springer-Verlag Berlin Heidelberg 2014 / Online publiziert: 31. Oktober 2014

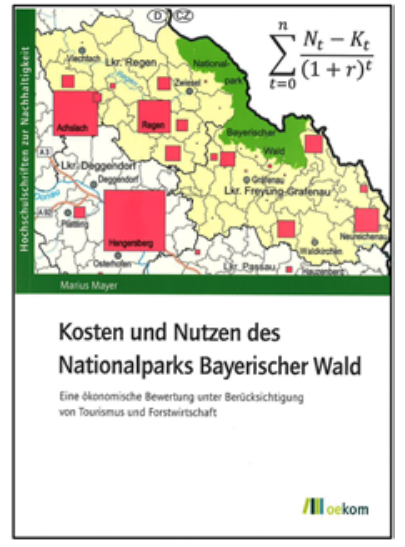

Der Nationalpark Bayerischer Wald - ältester in der Bundesrepublik - ist schon zu Beginn der 1980er Jahre Gegenstand ökonomischer Berechnungen gewesen, war doch das Motiv zu seiner Gründung eher ein ökonomisches als ein ökologisches: Er sollte zur Entwicklung des ,rückständigen' Grenzlandes des Freistaates beitragen, insbesondere durch die Förderung des Tourismus. Inzwischen sind auch im deutschsprachigen Bereich etliche Werke zur ökonomischen Bedeutung von Großschutzgebieten entstanden, die nun durch eine beeindruckende Studie von nahezu 600 Seiten ergänzt werden.

Prof. em. Dr. U. Hampicke $(\bowtie)$

Institut für Botanik und Landschaftsökologie,

Ernst-Moritz-Arndt Universität Greifswald,

Soldmannstraße 15,

17489 Greifswald, Deutschland

E-Mail: hampicke@uni-greifswald.de
Das Buch gliedert sich neben der Einleitung und der Schlussbetrachtung mit Ausblick in neun Kapitel, von denen vier (Kap. 2-5, S. 34-184) die Theorie der ökonomischen Bewertung sowie den Forschungsstand zur Bewertung von Nationalparken darlegen. Zwei weitere Kap. (6 und 7, S. 185-251) beschreiben das Untersuchungsgebiet und erläutern das empirische Forschungsdesign der Arbeit. Die Kap. 8-10 (S. 252-484) berechnen dann in allen Einzelheiten die Kosten und den Nutzen des Nationalparks und führen in einer Diskussion die Ergebnisse zusammen.

Es handelt sich beim vorliegenden Werk um eine Dissertationsschrift. In einer solchen Arbeit geht es nicht allein darum, dem Leser neue Erkenntnisse vorzulegen, vielmehr liegt hier in erster Linie eine akademische Prüfungsleistung vor, in der der Autor zeigen soll, was er alles weiß und kann. So muss der Leser darauf vorbereitet sein, in den theoretischen Teilen Passagen zu finden, die dem Experten längst geläufig sind, dem Nicht-Experten aber nicht unbedingt viel sagen, weil sie zu viel voraussetzen - es ist nun einmal kein Lehrbuch. Auch muss der Leser bei den Berechnungen des Autors eine Fülle von Zahlen und Fakten, Annahmen und Szenarien ertragen, was manchen Seufzer entlocken dürfte. Der Autor hat eine staunenswerte Arbeitsleistung erbracht und es ist ihm nicht zu verübeln, dass er diese ohne Abstriche dokumentieren möchte. Dem Charakter der Dissertationsschrift entspricht auch ein Apparat mustergültiger Verzeichnisse sowie der Beweis enzyklopädischer Belesenheit in einem Literaturverzeichnis von 43 (!) Seiten, wobei freilich manche Zeitungsnotiz und nicht nur diese entbehrlich wäre.

Dem Autor und mit ihm der Wirtschaftswissenschaft zufolge ist das in der Öffentlichkeit verbreitete Fehlurteil über den ökonomischen Wert von Großschutzgebieten der Tatsache geschuldet, dass ihre Kosten am Markt relativ leicht erhoben werden, ihr Nutzen jedoch, da es sich 
um Kollektivgüter handelt, schwer zu messen und leicht zu leugnen sind, so dass es Partikularinteressen (die sich gern als die ,Volksmeinung' gerieren) gelingt, insbesondere Nationalparke herabzusetzen oder gar ihre Gründung zu verhindern.

So nimmt die Erhebung des am Markt nur unzureichend oder gar nicht abgebildeten Nutzens insbesondere der Erholungssuchenden einen wichtigen Platz in der Arbeit ein, wozu es etablierte Techniken, wie die Reisekostenmethode (Travel Cost Method, TCM) und die Zahlungsbereitschaftserhebung mittels Befragung (Contigent Valuation Method, CVM) gibt. Allein diese Erhebung hätte schon eine Dissertationsschrift füllen können. Der Autor lässt es jedoch nicht dabei bewenden, sondern vergleicht die Wertschöpfung des Nationalparks auf dem Gebiet der Kollektivgüter mit der, die die wahrscheinlichste Alternative zu ihm hervorbringen würde, nämlich die überwiegend holzwirtschaftliche Nutzung durch den Bayerischen Staatsforstbetrieb. Unter Erhebung zahlreicher weiterer Kostenpositionen gelingt dem Autor daher eine volle Kosten-Nutzen-Analyse. Auch strebt er an zu ermitteln, wie sich Kosten und Nutzen räumlich, personell und institutionell verteilen, insbesondere wie viel vom Nutzen in der Region verbleibt.

Die theoretischen Teile (S. 34-184) sind im Allgemeinen gut, aber lassen gelegentlich die Kenntnis der relevantesten Literatur und eine sichere Urteilsfähigkeit vermissen. So werden unendlich viele Sekundär- und Tertiärautoren verarbeitet, nicht aber beim wichtigen Abschnitt über Property Rights (Kap. 2.2.5, S. 63 f.) die Nobelpreisträgerin Ostrom (1990) sowie Bromley (1997). Stattdessen nimmt der Autor immer noch Hardin (1968) ernst, dessen fatale Verwechslung von Gemeineigentum (mit Regeln) und Open Access (ohne Regeln) Generationen von Lesern verwirrt hat.

Gegen Kürzungen auf anderen Gebieten hätte es gelohnt, in die Theorie der Property Rights tiefer einzudringen. Auch die Ausführungen zur Diskontierung (S. 91 f.) überzeugen nicht, was sich im Ergebnisteil bei den Kapitalisierungen jährlicher Werte rächt. Der Autor kann natürlich nichts dafür, dass sein Werk in eine verwunderliche Epoche der Fast-Zinslosigkeit fällt, jedoch wären die von ihm gewählten Geschäftszinssätze von 5 und $10 \%$ pro Jahr auch unter normalen Umständen nicht als Diskontsätze zu rechtfertigen gewesen.

Manches in der Theorie ließe sich besser, kürzer und eleganter darstellen. Um nur ein Beispiel zu nennen: Es ist zweifelhaft, ob die Kollegen des Autors in der Wirtschaftsgeographie die Passagen zu Kompensationsvariation und Äquivalenzvariation (Kap. 3.2.3, S. 84-86) verstehen, wenn sie sich sie nicht schon vorher angeeignet haben. Hier wie auch an zahlreichen anderen Stellen hätte eine einfache Strichzeichnung viele Worte erspart und es wäre den Lesern wie Schuppen von den Augen gefallen, was gemeint ist. Der
Autor hätte stärkeren Gebrauch von der graphischen Darstellung machen sollen.

Eine besondere Stärke der Arbeit ist dagegen in ihrer Verhaftung in der realen Lebenswirklichkeit, ihrer Kompetenz auch in technischen Dingen und ihrer Liebe zum Detail zu sehen, wie es besonders im Kap. 6 (Vorstellung des Untersuchungsgebietes, S. 185-233), aber auch an vielen anderen Stellen zum Ausdruck kommt. Der Autor folgt nicht der Untugend der meisten ,reinen' Ökonomen, sich allein für die Parallelwelt der Modelle und statistischen Testmethoden zu interessieren, sondern informiert den Leser über alles, was im Bayerischen Wald wichtig ist, natürlich auch über Borkenkäfer und den Orkan Kyrill.

$\mathrm{Zu}$ den Kosten des Nationalparks zählen nicht nur seine unmittelbaren Verwaltungs- und Baukosten, sondern auch - am wichtigsten - seine Opportunitätskosten in Gestalt des Verzichtes auf Erlöse aus dem Holzverkauf und der Jagd. Hinzu kommen gewisse indirekte Kosten, unter anderem durch mögliche Schadwirkungen auf benachbarte Wirtschaftsforsten, hervorgerufen durch Borkenkäfer und Wildschweine. Die Erhebung des Nutzens erfolgt im Wesentlichen in drei Schritten: einer Wertschöpfungsanalyse, also der Erhebung der durch die Besucher induzierten Geldströme, einer Erhebung der Reisekosten mit der TCM und einer Zahlungsbereitschaftsanalyse mit der CVM. Empirische Grundlage ist eine aufwändige und reich strukturierte Besucherbefragung.

Die Ergebnisse werden differenziert in mehreren Szenarien vorgetragen. Sehr grob gesprochen erfährt der Leser, dass der Nationalpark in volkswirtschaftlicher Hinsicht eher etwas besser abschneidet als die Alternativnutzung durch reine Holzwirtschaft. Es ist nach den vorliegenden Berechnungen recht unwahrscheinlich, dass er eine ökonomische Fehlinvestition wäre. Das ist ein interessantes Ergebnis, welches zudem sehr sorgfältig mit anderen Werken in der Literatur verglichen wird.

Der Autor wendet den in der Regional- und Tourismusökonomie geläufigen Methodenmix an. Steht ihm das auch ohne Zweifel zu, so häufen sich für den Theoretiker allerdings Fragen. Das letzte Wort darüber, was echte Wertschöpfung und was Verlagerungseffekt ist, ist wohl noch längst nicht gesprochen. Die Touristen hätten ohne den Nationalpark ihr Geld auch irgendwo ausgegeben. In den Kapiteln zur TCM und CVM wird zwar so viel induktive Statistik und zugehöriger Jargon aufgefahren, dass dem Leser Hören und Sehen vergeht, aber die einfachsten Fragen bleiben offen. Die Frage in der CVM nach dem maximalen Eintrittspreis, den ein Besucher zu zahlen bereit wäre, ist natürlich in keiner Weise eine Erhebung von Nichtgebrauchswerten, wie es die Überschrift (S. 411) ankündigt. Die TCM erhebt die Marshallsche Konsumentenrente, die $\mathrm{CVM}$ in der vorliegenden Form theoretisch die Equivalent Variation. Beide sollten nicht allzu weit auseinander liegen, 
die Differenz wäre zu begründen. Das Ergebnis der TCM liegt zwischen 15 und 35 Mio. $€$ pro Jahr (S. 376), das der CVM dagegen unter 2 Mio. $€$ pro Jahr (S. 431). Vielleicht ist des Rätsels Lösung, dass die Befragten in der CVM gar nicht ihre Equivalent Variation angaben, sondern einen Tarif als Eintrittspreis, den sie als angemessen empfanden. Die Vermischung von Zahlungsströmen (Marktpreisen und Tarifen) und Konsumentenrenten ist problematisch. Wenn beim Nutzen die Konsumentenrenten der Besucher zählen, dann dürften bei den Kosten nicht die Tarife für die Jagdpacht, sondern müssten auch die viel höheren entgangenen Konsumentenrenten der Jäger erhoben werden.

Trotz dieser Einwände handelt es sich um eine sehr wertvolle Arbeit, die alle Akteure im Bereich der Großschutzgebiete wahrnehmen sollten. Besonders im abschließenden Kap. 10.2 (Diskussion der Ergebnisse, S. 455-479) demonstriert der Autor sein in langjähriger Erfahrung gewonnenes, auch nicht-ökonomisches Fachwissen und kann alle Verlautbarungen von Gegnern des Nationalparks, denen kein Argument zu verstiegen ist, wenn es nur ihren Interessen dient, kontern. Sprache und Aufmachung des Buches sind angenehm, allerdings fehlt ein Index. Leider klaffen Umfang des Buches und heutiges Leseverhalten besonders studierender junger Leute weit auseinander, so dass dem Autor zu raten ist, seine Ergebnisse auch in kürzerer Form in Fachzeitschriften zu veröffentlichen.

\section{Literatur}

Bromley, D.W. (1997): Property regimes in environmental economics. In: Folmer, H.; Tietenberg, T. (Hrsg.): The International Yearbook of Environmental and Resource Economics 1997/98. Cheltenham, $1-27$.

Hardin, G. (1968): The tragedy of the commons. Science 13 (162), $1243-1248$.

Ostrom, E. (1990): Governing the Commons. The Evolution of Institutions for Collective Action. Cambridge. 